\title{
Convergence rates in regularization for a system of nonlinear ill-posed equations with $m$-accretive operators
}

Jong Kyu Kim ${ }^{1 *}$ and Nguyen Buong ${ }^{2}$

\section{"Correspondence:}

jongkyuk@kyungnam.ac.kr

${ }^{1}$ Department of Mathematics

Education, Kyungnam University,

Changwon, 631-701, Gyeongnam

Full list of author information is

available at the end of the article

\begin{abstract}
In this paper, we study an operator version of the modified Browder-Tikhonov regularization method for finding a common solution for a system of ill-posed operator equations involving $m$-accretive operators $A_{i}, i=0, \ldots, N$, in a reflexive Banach space. The convergence rates of the regularized solutions are estimated not only in the infinite-dimensional space, but also in connection with its finite-dimensional approximations without the weakly sequential continuity of the dual mapping.
\end{abstract}

MSC: $47 \mathrm{H} 17 ; 47 \mathrm{H} 20$

Keywords: accretive operators; demicontinuous; convex Banach space; modified Browder-Tikhonov regularization; Fréchet differentiability

\section{Introduction}

Let $X$ be a real reflexive Banach space with the property of approximations and its dual space $X^{*}$ be strictly convex. The norms of $X$ and $X^{*}$ are denoted by the symbol $\|\cdot\|$. We write $\left\langle x, x^{*}\right\rangle$ instead of $x^{*}(x)$ for $x^{*} \in X^{*}$ and $x \in X$.

Definition 1.1 A Banach space $X$ is said to be strictly convex if for $x, y \in S_{X}$ with $x \neq y$, then

$$
\|(1-\lambda) x+\lambda y\|<1 \quad \forall \lambda \in(0,1)
$$

where $S_{X}$ is the unit sphere $S_{X}=\{x \in X:\|x\|=1\}$.

Definition 1.2 A mapping $j$ from $X$ onto $X^{*}$ is called the normalized dual mapping of $X$, if it satisfies the condition

$$
\langle x, j(x)\rangle=\|x\|^{2}, \quad\|j(x)\|=\|x\| \quad \forall x \in X .
$$

It is well known that if $X^{*}$ is strictly convex then $j$ is single-valued.

Definition 1.3 An operator $A$ from $X$ to $X$ is said to be accretive, if

$$
\langle A(x)-A(y), j(x-y)\rangle \geq 0 \quad \forall x, y \in D(A)
$$

O2014 Kim and Buong; licensee Springer. This is an Open Access article distributed under the terms of the Creative Commons Attribution License (http://creativecommons.org/licenses/by/2.0), which permits unrestricted use, distribution, and reproduction in any medium, provided the original work is properly cited. 
where $D(A)$ denotes the domain of $A$. An accretive operator $A$ is said to be an $m$-accretive, if $\mathcal{R}(A+\lambda I)=X$ for $\lambda>0$ where $\mathcal{R}(A)$ and $I$ denote the range of $A$ and the identity mapping of $X$, respectively.

Definition 1.4 An operator $A$ from $X$ to $X$ is said to be

(i) demicontinuous if $x_{n} \rightarrow x$ in $X$ implies $A\left(x_{n}\right) \rightarrow A(x)$,

(ii) weakly continuous if $x_{n} \rightarrow x$ implies $A\left(x_{n}\right) \rightarrow A(x)$.

It is well known that if $A$ is accretive, and is continuous, demicontinuous, or weakly continuous, then it is $m$-accretive [1-3].

Definition 1.5 A mapping $A$ from $X$ to $X$ is called Fréchet differentiable at a point $x \in$ $D(A)$, if

$$
A(x+h)-A(x)=B(x) h+o(\|h\|) \quad \forall x+h \in D(A)
$$

where $B(x)$ is a bounded linear mapping from $X$ to $X$. And the Fréchet derivative of $A$ at $x \in D(A)$ is denoted by $A^{\prime}(x)$.

Let $\left\{A_{i}\right\}_{i=0}^{N}$ be a family of $N+1$ accretive operators in $X$ and satisfy one of the above mentioned three continuities.

Our problem is to find a common solution of the following operator equations:

$$
A_{i}(x)=f_{i}, \quad f_{i} \in \mathcal{R}\left(A_{i}\right), i=0, \ldots, N .
$$

Set

$$
S=\bigcap_{i=0}^{N} S_{i}
$$

where $S_{i}$ is the solution set of (1.1), that is, $S_{i}=\left\{x: A_{i}(x)=f_{i}\right\}$.

Suppose that $S \neq \emptyset$.

For $m$-accretive operators, some results of the approximating solution for each equation in (1.1) under suitable different conditions are investigated in [4-10], and [11].

The system of equations (1.1) is ill-posed, because each one of the system is ill-posed. By ill-posedness, we mean that its solutions do not depend continuously on the data $\left(A_{i}, f_{i}\right)$. Therefore, we have to use the stable methods in order to solve the problem. Some stable methods of approximating solution for each equation in (1.1) with $m$-accretive operator are investigated in [12,13], and [14] having the weakly sequentially continuous duality mapping $j$. In [15-19], the authors considered the modified Browder-Tikhonov regularization method with the regularization parameter choice without the property for $j$, for the case of demicontinuous or weakly continuous accretive operators $A_{i}$ satisfying the condition

$$
\left\|A_{i}(y)-A_{i}\left(x_{0}^{i}\right)-j^{*} A_{i}^{\prime}\left(x_{0}^{i}\right) j\left(y-x_{0}^{i}\right)\right\| \leq \tilde{\tau}\left\|y-x_{0}^{i}\right\|\left\|A_{i}^{\prime}\left(x_{*}\right) j\left(y-x_{0}^{i}\right)\right\|
$$

for $y$ in some neighborhood of $S_{i}$, where $A_{i}^{\prime}\left(x_{0}^{i}\right)$ is the Fréchet derivative of $A_{i}$ at $x_{0}^{i} \in S_{i}$, $\tilde{\tau}$ is some positive constant, and $j^{*}$ is the normalized duality mapping of $X^{*}$. 
In many papers, for each $i$, the regularized solution of (1.1) is constructed by the following operator equation:

$$
A_{i}^{h}(x)+\alpha x=f_{i}^{\delta},
$$

where $\left(A_{i}^{h}, f_{i}^{\delta}\right)$ is the approximation of $\left(A_{i}, f_{i}\right)$ satisfying the conditions:

$$
\left\|A_{i}^{h}(x)-A_{i}(x)\right\| \leq h g(\|x\|), \quad\left\|f_{i}-f_{i}^{\delta}\right\| \leq \delta, \quad h, \delta \rightarrow 0,
$$

$g(t)$ is a nonnegative bounded (image of bounded set is bounded) real function, and $A_{i}^{h}$ is also accretive and the same continuity as $A_{i}$.

The system of equations (1.1) can be written in the form

$$
\mathcal{A}(x)=f,
$$

where $\mathcal{A}: \mathcal{X} \rightarrow \mathcal{X}:=X \times \cdots \times X$ is defined by $\mathcal{A}(x):=\left(A_{0}(x), \ldots, A_{N}(x)\right)$, and $f:=$ $\left(f_{0}, \ldots, f_{N}\right)$.

Note that (1.4) can be seen as a special case of (1.1) with $N=0$. However, one potential advantage of (1.1) over (1.4) can be that it might better reflect the structure of the underlying information $\left(f_{0}, \ldots, f_{N}\right)$ leading to the couplet system, than a plain concatenation into one single data element $f$ could. In particular, the second advantage is that in estimating convergence rates of regularization solution, which is showed later, we need only the smooth property for one among $A_{i}$, while for (1.4) we need the property for $A_{i}, i=0, \ldots, N$.

When for each $i, A_{i}$ is the nonlinear Fréchet differentiable operator from the Hilbert space $X$ to the Hilbert space $Y_{i}$ with derivative being uniformly bounded in a neighborhood of a common solution, a stable method for problem (1.1) is considered in [20].

In this paper, we show that a common solution of (1.1) involving $m$-accretive operators $A_{i}$, without the weakly sequentially continuous property of $j$, can be approximated by the modified Browder-Tikhonov regularization method which is described by the following operator equation:

$$
A_{0}^{h}(x)+\alpha^{1+\mu_{0}} \sum_{i=1}^{N}\left(A_{i}^{h}(x)-f_{i}^{\delta}\right)+\alpha x=f_{0}^{\delta}, \quad \mu_{0} \geq 0,
$$

where $\alpha>0$ is a small regularization parameter. Since the operator

$$
A_{0}^{h}+\alpha^{1+\mu_{0}} \sum_{i=1}^{N}\left(A_{i}^{h}-f_{i}^{\delta}\right)
$$

has the same properties as each $A_{i}^{h}$, it is also $m$-accretive. Therefore, (1.5) has a unique solution denoted by $x_{\alpha}^{\tau}, \tau=(\delta, h)$, for every value $\alpha>0$.

In the following section, the convergence rates of the regularized solution $x_{\alpha}^{\tau}$ and its finite-dimensional approximations $x_{\alpha, n}^{\tau}$ are established under an assumption similar to (1.2).

The symbols ' $\rightarrow$ ' and ' $\rightarrow$ ' denote strong and weak convergence, respectively, and the notation $a \sim b$ means that $a=o(b)$ and $b=o(a)$. 


\section{Main results}

Assumption A There exists a constant $\tau_{0}>0$ such that

$$
\left\|A_{0}(x)-A_{0}\left(x_{0}\right)-j^{*} A_{0}^{\prime}\left(x_{0}\right)^{*} j\left(x-x_{0}\right)\right\| \leq \tau_{0}\left\|A_{0}(x)-A_{0}\left(x_{0}\right)\right\|, \quad \forall x \in X .
$$

Now, we are in a position to introduce the main theorem.

Theorem 2.1 Let $X$ be a real reflexive Banach space with the property of approximations and its dual space $X^{*}$ be strictly convex. Let $\left\{A_{i}\right\}_{i=0}^{N}$ be a family of $N+1$ accretive operators in $X$ and satisfy one of the above mentioned continuities. Assume that the following conditions hold:

(i) $A_{0}$ is Fréchet differentiable at $x_{0}$ with Assumption A.

(ii) There exists an element $z \in X$ such that

$$
A_{0}^{\prime}\left(x_{0}\right) z=-x_{0}
$$

(iii) The parameter $\alpha$ is chosen such that $\alpha \sim(\delta+h)^{\mu}, 0<\mu<1$.

Then, for $0<\delta+h<1$, we have

$$
\left\|x_{\alpha}^{\tau}-x_{0}\right\|=o\left((\delta+h)^{\theta}\right), \quad \theta=\min \{1-\mu, \mu / 2\}
$$

Proof From the property of $j, A_{i}^{h},(1.1),(1.3),(1.5)$, and condition (ii), it follows that

$$
\begin{aligned}
\left\|x_{\alpha}^{\tau}-x_{0}\right\|^{2}= & \left\langle x_{\alpha}^{\tau}-x_{0}, j\left(x_{\alpha}^{\tau}-x_{0}\right)\right\rangle \\
= & \frac{1}{\alpha}\left\langle f_{0}^{\delta}-A_{0}^{h}\left(x_{\alpha}^{\tau}\right)+\alpha^{1+\mu_{0}} \sum_{i=1}^{N}\left(f_{i}^{\delta}-A_{i}^{h}\left(x_{\alpha}^{\tau}\right)\right)-\alpha x_{0}, j\left(x_{\alpha}^{\tau}-x_{0}\right)\right\rangle \\
\leq & \frac{1+N \alpha^{1+\mu_{0}}}{\alpha}\left[\delta+h g\left(\left\|x_{0}\right\|\right)\right]\left\|x_{\alpha}^{\tau}-x_{0}\right\| \\
& +\left\langle z, A_{0}^{\prime}\left(x_{0}\right)^{*} j\left(x_{\alpha}^{\tau}-x_{0}\right)\right\rangle .
\end{aligned}
$$

Therefore, $\left\{x_{\alpha}^{\tau}\right\}$ is a bounded set. Since

$$
\left\langle z, A_{0}^{\prime}\left(x_{0}\right)^{*} j\left(x_{\alpha}^{\tau}-x_{0}\right)\right\rangle \leq\|z\|\left\|A_{0}^{\prime}\left(x_{0}\right)^{*} j\left(x_{\alpha}^{\tau}-x_{0}\right)\right\|,
$$

by virtue of Assumption A, we have

$$
\begin{aligned}
& \left\|A_{0}^{\prime}\left(x_{0}\right)^{*} j\left(x_{\alpha}^{\tau}-x_{0}\right)\right\| \\
& \quad=\left\|j^{*} A_{0}^{\prime}\left(x_{0}\right)^{*} j\left(x_{\alpha}^{\tau}-x_{0}\right)\right\| \\
& \quad \leq\left(\tau_{0}+1\right)\left\|A_{0}\left(x_{\alpha}^{\tau}\right)-f_{0}\right\| \\
& \quad \leq\left(\tau_{0}+1\right)\left[\left\|A_{0}^{h}\left(x_{\alpha}^{\tau}\right)-f_{0}^{\delta}\right\|+\delta+h g\left(\left\|x_{\alpha}^{\delta}\right\|\right)\right] \\
& \quad \leq\left(\tau_{0}+1\right)\left[\alpha^{1+\mu_{0}} \sum_{i=1}^{N}\left\|A_{i}^{h}\left(x_{\alpha}^{\tau}\right)-f_{i}^{\delta}\right\|+\alpha\left\|x_{\alpha}^{\delta}\right\|+\delta+h g\left(\left\|x_{\alpha}^{\delta}\right\|\right)\right] .
\end{aligned}
$$


Since $\alpha \sim(\delta+h)^{\mu}, 0<\mu<1$, and $g(t)$ is a bounded function, from (2.1) and the last inequality, we obtain

$$
\left\|x_{\alpha}^{\tau}-x_{0}\right\|^{2} \leq C_{1}(\delta+h)^{1-\mu}\left\|x_{\alpha}^{\tau}-x_{0}\right\|+C_{2}(\delta+h)^{\mu}, \quad 0<\delta+h<1,
$$

where $C_{1}$ and $C_{2}$ are positive constants. Now, by using the implication

$$
a, b, c \geq 0, p>q, \quad a^{p} \leq b a^{q}+c \quad \Longrightarrow \quad a^{p}=o\left(b^{p /(p-q)}+c\right),
$$

we obtain

$$
\left\|x_{\alpha}^{\tau}-x_{0}\right\|=o\left((\delta+h)^{\theta}\right), \quad \theta=\min \{1-\mu, \mu / 2\}
$$

This completes the proof.

Now, we consider the problem of approximating (1.5) by the sequence of finitedimensional problems

$$
A_{0, n}^{h}(x)+\alpha^{1+\mu_{0}} \sum_{i=1}^{N}\left(A_{i, n}^{h}(x)-f_{i, n}^{\delta}\right)+\alpha x=f_{0, n}^{\delta}, \quad x \in X_{n},
$$

where $f_{i, n}^{\delta}=P_{n} f_{i}^{\delta}, A_{i, n}^{h}=P_{n} A_{i}^{h} P_{n}, P_{n}$ is the linear projection from $X$ onto $X_{n}, P_{n} x \rightarrow x$ for all $x \in X,\left\|P_{n}\right\| \leq C_{0}, C_{0}$ is some positive constant, and $\left\{X_{n}\right\}$ is the sequence of finitedimensional subspaces of $X$ such that

$$
X_{1} \subset X_{2} \subset \cdots \subset X
$$

It is easy to see that $A_{i, n}^{h}$ are also $m$-accretive. The aspects of existence and convergence of the solution $x_{\alpha, n}^{\tau}$ of problem (2.2), as $n \rightarrow \infty$, to the solution $x_{\alpha}^{\tau}$ of the operator equation (1.5) for each $\alpha>0$ has been studied in [21]. The question under which conditions the sequence $\left\{x_{\alpha, h}^{\tau}\right\}$ converges to a solution $x_{0}$, as $\alpha, \delta, h \rightarrow 0$ and $n \rightarrow \infty$, and the convergence rates of $\left\{x_{\alpha, n}^{\tau}\right\}$ are subject of our further investigations.

In addition, suppose that $j$ satisfies the following inequality:

$$
\|j(x)-j(y)\| \leq C(R)\|x-y\|^{v}, \quad 0<v<1,
$$

where $C(R), R>0$ is positive increasing function on $R=\max \{\|x\|,\|y\|\}$ (see [11]).

Set

$$
\gamma_{n}=\left\|\left(I-P_{n}\right) x_{0}\right\|
$$

Theorem 2.2 Let $X$ be a real reflexive Banach space with the property of approximations and its dual space $X^{*}$ be strictly convex. Let $\left\{A_{i}\right\}_{i=0}^{N}$ be a family of $N+1$ accretive operators in $X$ and satisfy one of the above mentioned continuities. Suppose that the following conditions hold:

(i) $A_{0}$ is Fréchet differentiable with Assumption $\mathrm{A}$ and the derivative $A_{0}^{\prime}$ being uniformly bounded at $x_{0}$. 
(ii) There exists an element $z \in X$ such that

$$
A_{0}^{\prime}\left(x_{0}\right) z=-x_{0}
$$

(iii) The parameter $\alpha$ is chosen such that $\alpha \sim\left(\delta+h+\gamma_{n}\right)^{\mu}, 0<\mu<1$.

Then, for $0<\delta+h<1$, we have

$$
\left\|x_{\alpha, n}^{\tau}-x_{0}\right\|=o\left(\left(\delta+h+\gamma_{n}\right)^{\theta}+\gamma_{n}^{v / 2}\right), \quad \theta=\min \{1-\mu, \mu / 2\}
$$

Proof Set $x_{0}^{n}=P_{n} x_{0}$. From (2.2) and the property $j^{n}(x)=j(x)$ for all $x \in X_{n}$, where $j^{n}=P_{n}^{*} j P_{n}$ is the dual mapping of $X_{n}$ (see [13]), it follows that

$$
\begin{aligned}
\left\|x_{\alpha, n}^{\tau}-x_{0}^{n}\right\|^{2}= & \left\langle x_{\alpha, n}^{\tau}-x_{0}^{n}, j^{n}\left(x_{\alpha, n}^{\tau}-x_{0}^{n}\right)\right\rangle \\
= & \frac{1}{\alpha}\left\langle f_{0, n}^{\delta}-A_{0, n}^{h}\left(x_{\alpha, n}^{\tau}\right), j^{n}\left(x_{\alpha, n}^{\tau}-x_{0}^{n}\right)\right\rangle \\
& +\left\langle-x_{0}^{n}, j^{n}\left(x_{\alpha, n}^{\tau}-x_{0}^{n}\right)\right\rangle \\
& +\alpha^{\mu_{0}} \sum_{i=1}^{N}\left\langle f_{i, n}^{\delta}-A_{i, n}^{h}\left(x_{\alpha, n}^{\tau}\right), j^{n}\left(x_{\alpha, n}^{\tau}-x_{0}^{n}\right)\right\rangle .
\end{aligned}
$$

Clearly,

$$
\begin{aligned}
\left\langle f_{0, n}^{\delta}\right. & \left.-A_{0, n}^{h}\left(x_{\alpha, n}^{\tau}\right), j^{n}\left(x_{\alpha, n}^{\tau}-x_{0}^{n}\right)\right\rangle \\
= & \left\langle f_{0}^{\delta}-f_{0}+A_{0}\left(x_{0}\right)-A_{0}\left(x_{0}^{n}\right)+A_{0}\left(x_{0}^{n}\right)-A_{0}^{h}\left(x_{0}^{n}\right), j^{n}\left(x_{\alpha, n}^{\tau}-x_{0}^{n}\right)\right\rangle \\
& +\left\langle A_{0}^{h}\left(x_{0}^{n}\right)-A_{0}^{h}\left(x_{\alpha, n}^{\tau}\right), j^{n}\left(x_{\alpha, n}^{\tau}-x_{0}^{n}\right)\right\rangle \\
\leq & {\left[\delta+\left\|A_{0}\left(x_{0}\right)-A_{0}\left(x_{0}^{n}\right)\right\|+h g\left(\left\|x_{0}^{n}\right\|\right)\right]\left\|x_{\alpha, n}^{\tau}-x_{0}^{n}\right\| . }
\end{aligned}
$$

Due to condition (i) and $x_{0}^{n} \rightarrow x_{0}$ as $n \rightarrow \infty$, we have

$$
\left\|A_{0}\left(x_{0}^{n}\right)-A_{0}\left(x_{0}\right)\right\| \leq C_{0}^{\prime} \gamma_{n}
$$

where $C_{0}^{\prime}$ is a positive constant such that

$$
\left\|A_{0}^{\prime}\left(x_{0}\right)\right\| \leq C_{0}^{\prime}
$$

for $x$ in a neighborhood of $x_{0}$. Thus, we have

$$
\left\langle f_{0, n}^{\delta}-A_{0, n}^{h}\left(x_{\alpha, n}^{\tau}\right), j^{n}\left(x_{\alpha, n}^{\tau}-x_{0}^{n}\right)\right\rangle \leq\left[\delta+h g\left(\left\|x_{0}^{n}\right\|\right)+C_{0}^{\prime} \gamma_{n}\right]\left\|x_{\alpha, n}^{\tau}-x_{0}^{n}\right\| .
$$

Each term of the sum in (2.4) is estimated as follows:

$$
\begin{aligned}
\left\langle f_{i, n}^{\delta}\right. & \left.-A_{i, n}^{h}\left(x_{\alpha, n}^{\tau}\right), j^{n}\left(x_{\alpha, n}^{\tau}-x_{0}^{n}\right)\right\rangle \\
& =\left\langle f_{i}^{\delta}-A_{i}^{h}\left(x_{\alpha, n}^{\tau}\right), j^{n}\left(x_{\alpha, n}^{\tau}-x_{0}^{n}\right)\right\rangle \\
& =\left\langle f_{i}^{\delta}-A_{i}^{h}\left(x_{0}^{n}\right)+A_{i}^{h}\left(x_{0}^{n}\right)-A_{i}^{h}\left(x_{\alpha, n}^{\tau}\right), j^{n}\left(x_{\alpha, n}^{\tau}-x_{0}^{n}\right)\right\rangle
\end{aligned}
$$




$$
\begin{aligned}
& \leq\left\langle f_{i}^{\delta}-A_{i}^{h}\left(x_{0}^{n}\right), j^{n}\left(x_{\alpha, n}^{\tau}-x_{0}^{n}\right)\right\rangle \\
& \leq\left[\delta+h g\left(\left\|x_{0}^{n}\right\|\right)+\left\|A_{i}\left(x_{0}\right)-A_{i}\left(x_{0}^{n}\right)\right\|\right]\left\|x_{\alpha, n}^{\tau}-x_{0}^{n}\right\| .
\end{aligned}
$$

By virtue of the continuity of $A_{i}$, there exists a positive constant $C^{\prime}$ such that

$$
\left\|A_{i}\left(x_{0}\right)-A_{i}\left(x_{0}^{n}\right)\right\| \leq C^{\prime}, \quad i=1, \ldots, N .
$$

From (2.4)-(2.6), we see that

$$
\begin{aligned}
\left\|x_{\alpha, n}^{\tau}-x_{0}^{n}\right\|^{2} \leq & {\left[\frac{1}{\alpha}\left(\delta+h+C_{0}^{\prime} \gamma_{n}\right)+\alpha^{\mu_{0}}\left(\delta+h g\left(\left\|x_{0}^{n}\right\|\right)+C^{\prime}\right)\right]\left\|x_{\alpha, n}^{\tau}-x_{0}^{n}\right\| } \\
& +\left\langle-x_{0}, j\left(x_{\alpha, n}^{\tau}-x_{0}^{n}\right)\right\rangle .
\end{aligned}
$$

Consequently, $\left\{x_{\alpha, n}^{\tau}\right\}$ is bounded as $\delta, h, \alpha \rightarrow 0$ and $n \rightarrow \infty$. Obviously, from (2.3), Assumption $\mathrm{A}$, and condition (ii), it follows that

$$
\begin{aligned}
\left\langle-x_{0}, j\left(x_{\alpha, n}^{\tau}-x_{0}^{n}\right)\right\rangle & =\left\langle z, A_{0}^{\prime}\left(x_{0}\right)^{*}\left[j\left(x_{\alpha, n}^{\tau}-x_{0}^{n}\right)-j\left(x_{\alpha, n}^{\tau}-x_{0}\right)\right]\right\rangle+\left\langle z, A_{0}^{\prime}\left(x_{0}\right)^{*} j\left(x_{\alpha, n}^{\tau}-x_{0}\right)\right\rangle \\
& \leq C\left(R_{1}\right)\left\|A_{0}^{\prime}\left(x_{0}\right)^{*}\right\|\|z\| \gamma_{n}^{v}+\|z\|\left\|A_{0}^{\prime}\left(x_{0}\right)^{*} j\left(x_{\alpha, n}^{\tau}-x_{0}\right)\right\|,
\end{aligned}
$$

where $R_{1}$ is a positive constant with $R_{1} \geq \max \left\{\left\|x_{0}\right\|,\left\|x_{\alpha, n}^{\tau}\right\|\right\}$.

On the other hand,

$$
\begin{aligned}
\left\|A_{0}^{\prime}\left(x_{0}\right)^{*} j\left(x_{\alpha, n}^{\tau}-x_{0}\right)\right\| & \leq\left(\tau_{0}+1\right)\left\|A_{0}\left(x_{\alpha, n}^{\tau}\right)-f_{0}\right\| \\
& \leq\left(\tau_{0}+1\right)\left[\left\|A_{0}^{h}\left(x_{\alpha, n}^{\tau}\right)-f_{0}^{h}\right\|+\delta+h g\left(\left\|x_{\alpha, n}^{\tau}\right\|\right)\right] .
\end{aligned}
$$

By virtue of the Hahn-Banach theorem, there exists an element $y^{*} \in X^{*}$ with $\left\|y^{*}\right\|=1$ such that

$$
\left\|A_{0}^{h}\left(x_{\alpha, n}^{\tau}\right)-f_{0}^{h}\right\|=\left\langle A_{0}^{h}\left(x_{\alpha, n}^{\tau}\right)-f_{0}^{h}, y^{*}\right\rangle .
$$

Since

$$
\left\langle A_{0}^{h}\left(x_{\alpha, n}^{\tau}\right)-f_{0}^{h}, y^{*}\right\rangle=\left\langle A_{0}^{h}\left(x_{\alpha, n}^{\tau}\right)-f_{0}^{h},\left(I^{*}-P_{n}^{*}\right) y^{*}\right\rangle+\left\langle A_{0}^{h}\left(x_{\alpha, n}^{\tau}\right)-f_{0}^{h}, P_{n}^{*} y^{*}\right\rangle
$$

and

$$
\left\|\left(I^{*}-P_{n}^{*}\right) y^{*}\right\| \leq 1 / 2
$$

for sufficiently large $n$, where $I^{*}$ is the identity operator in $X^{*}$, we have

$$
\left\|A_{0}^{h}\left(x_{\alpha, n}^{\tau}\right)-f_{0}^{h}\right\| \leq 2\left\|A_{0, n}^{h}\left(x_{\alpha, n}^{\tau}\right)-f_{0, n}^{h}\right\| .
$$


Therefore,

$$
\begin{aligned}
\left\|A_{0}^{\prime}\left(x_{0}\right)^{*} j\left(x_{\alpha, n}^{\tau}-x_{0}\right)\right\| \leq & \left(\tau_{0}+1\right) C_{0}\left[\alpha^{1+\mu_{0}} \sum_{i=1}^{N}\left\|A_{i}^{h}\left(x_{\alpha, n}^{\tau}\right)-f_{i}^{\delta}\right\|\right. \\
& \left.+\alpha\left\|x_{\alpha, n}^{\tau}\right\|+\delta+h g\left(\left\|x_{\alpha, n}^{\tau}\right\|\right)\right] .
\end{aligned}
$$

Thus, (2.7) has the form

$$
\left\|x_{\alpha, n}^{\tau}-x_{0}^{n}\right\|^{2} \leq \tilde{C}_{1}\left(\delta+h+\gamma_{n}\right)^{1-\mu}\left\|x_{\alpha, n}^{\tau}-x_{0}^{n}\right\|+\tilde{C}_{2}\left[\left(\delta+h+\gamma_{n}\right)^{\mu}+\gamma_{n}^{v}\right],
$$

where $\tilde{C}_{i}>0(i=1,2)$. Consequently, we have

$$
\left\|x_{\alpha, n}^{\tau}-x_{0}\right\|=O\left(\left(\delta+h+\gamma_{n}\right)^{\theta}+\gamma_{n}^{v / 2}\right), \quad \theta=\min \{1-\mu, \mu / 2\} .
$$

\section{This completes the proof.}

\section{Competing interests}

The authors declare that they have no competing interests.

\section{Authors' contributions}

The main idea of this paper was proposed by JKK. JKK and NB prepared the manuscript initially and performed all the steps of proof in this research. All authors read and approved the final manuscript.

\section{Author details}

'Department of Mathematics Education, Kyungnam University, Changwon, 631-701, Gyeongnam. ${ }^{2}$ Institute of Information Technology, Vietnamese Academy of Science and Technology, 18, Hoang Quoc Viet, q. Cau Giay, Ha Noi, Vietnam.

\section{Acknowledgements}

This work was supported by Basic Science Research Program through the National Research Foundation of Korea (NRF) funded by the Ministry of Education, Science and Technology (2012R1A1A2042138).

Received: 18 April 2014 Accepted: 21 October 2014 Published: 03 Nov 2014

\section{References}

1. Browder, FE: Nonlinear mapping of nonexpansive and accretive type in Banach spaces. Bull. Am. Math. Soc. 73, 875-882 (1967)

2. Fitzgibbon, WE: Weak continuous accretive operators. Bull. Am. Math. Soc. 79, 473-474 (1973)

3. Martin, RH Jr:: A global existence theorem for autonomous differential equations in Banach spaces. Proc. Am. Math. Soc. 26, 307-314 (1970)

4. Aoyama, $\mathrm{K}$, liduka, $\mathrm{H}$, Takahashi, W: Weak convergence of an iterative sequence for accretive operators in Banach spaces. Fixed Point Theory Appl. 2006, Article ID 35390 (2006)

5. Chen, R, Zhu, Z: Viscosity approximation fixed points for nonexpansive and $m$-accretive operators. Fixed Point Theory Appl. 2006, Article ID 81325 (2006)

6. Chidume, $\mathrm{CE}$, Zegeye, $\mathrm{H}$ : Iterative solution of $0 \in A x$ for an $m$-accretive operator $A$ in certain Banach spaces. J. Math. Anal. Appl. 269, 421-430 (2002)

7. Kim, JK: Convergence of Ishikawa iterative sequence for accretive Lipschitzian mappings in Banach spaces. Taiwan. J. Math. 10, 553-561 (2006)

8. Kim, JK, Tuyen, TM: Regularization proximal point algorithm for finding a common fixed point of a finite family of nonexpansive mappings in Banach spaces. Fixed Point Theory Appl. (2011). doi:10.1186/1687-1812-2011-52

9. Kim, JK, Buong, N: Regularization inertial proximal point algorithm for monotone hemicontinuous mapping and inverse strongly monotone mappings in Hilbert spaces. J. Inequal. Appl. 2010, Article ID 451916 (2010)

10. Miyake, $\mathrm{H}$, Takahashi, W: Approximating zero points of accretive operators with compact domains in general Banach spaces. Fixed Point Theory Appl. 2005(1), 93-102 (2005)

11. Moudafi, A: A remark on recent results for finding zeros of accretive operators. J. Appl. Math. Stoch. Anal. 2006, Article ID 56704 (2006)

12. Al'ber, Yl: On solution by the method of regularization for operator equation of the first kind involving accretive mappings in Banach spaces. Differ. Equ. 11, 2242-2248 (1975) (in Russian) 
13. Ryazanseva, IP: On nonlinear operator equations involving accretive mappings. Izv. Vysš. Učebn. Zaved., Mat. 1, 42-46 (1985) (in Russian)

14. Ryazantseva, IP: Regularization proximal algorithm for nonlinear equations of monotone type in Banach space. Zh. Vychisl. Mat. Mat. Fiz. 42, 1295-1303 (2002) (in Russian)

15. Buong, $\mathrm{N}$ : Convergence rates in regularization for nonlinear ill-posed equations under accretive perturbations. Zh. Vychisl. Mat. Mat. Fiz. 44, 397-402 (2004)

16. Buong, N: On nonlinear ill-posed accretive equations. Southeast Asian Bull. Math. 28, 595-600 (2004)

17. Buong, N: Generalized discrepancy principle and ill-posed equations involving accretive operators. Nonlinear Funct. Anal. Appl. 9, 73-78 (2004)

18. Buong, N, Hung, VQ: Newton-Kantorovich iterative regularization for nonlinear ill-posed equations involving accretive operators. Ukr. Mat. Zh. 57, 271-276 (2005)

19. Buong, N, Phuong, NTH: Regularization methods for nonlinear ill-posed equations involving $m$-accretive mappings in Banach spaces. Russ. Math. (Izv. VUZ) 57(2), 58-64 (2013)

20. Burger, M, Katenbacher, B: Regularizing Newton-Kaczmarz methods for nonlinear ill-posed problems. SIAM J. Numer. Anal. 44, 153-182 (2006)

21. Vainberg, MM: Variational Method and Method of Monotone Operators. Nauka, Moscow (1972) (in Russian); Wiley (1974) Translated from Russian by A. Libin, Translation edited by D. Louvish

$10.1186 / 1029-242 X-2014-440$

Cite this article as: Kim and Buong: Convergence rates in regularization for a system of nonlinear ill-posed equations with $m$-accretive operators. Journal of Inequalities and Applications 2014, 2014:440

\section{Submit your manuscript to a SpringerOpen ${ }^{\circ}$ journal and benefit from:}

- Convenient online submission

Rigorous peer review

- Immediate publication on acceptance

- Open access: articles freely available online

- High visibility within the field

- Retaining the copyright to your article 